\title{
Seleção de Soluções Circulares Para Resíduos Pré-Consumo da Indústria CalÇAdista Utilizando Níveis de Maturidade TeCnOlógica
}

Nicole Sofia Röhsig López (nicole.rohsig@gmail.com) - Universidade Federal do Rio Grande do Sul (UFRGS)

Istefani Carísio de Paula (istefani@producao.ufrgs.br) - Universidade Federal do Rio Grande do Sul (UFRGS)

\section{RESUMO}

A economia circular (EC) mostra-se cada vez mais importante. Há poucos estudos na indústria calçadista relativos aos resíduos têxteis, menos ainda os provenientes da produção de calçados. Estima-se que foram geradas 7.556 toneladas de resíduo têxtil nesta indústria no Brasil, 2020, revelando a importância de soluções para esses resíduos evitando problemas ambientais. O objetivo deste estudo é encontrar as soluções mais adequadas aos resíduos têxteis pré-consumo da indústria calçadista. Foi implementada pesquisa bibliográfica e pesquisa Desk. As estratégias circulares para o resíduo têxtil foram classificadas segundo os ciclos da EC e segundo a avaliação do nível de maturidade Technology Readiness Level (TRL). Principais resultados: (i) mapeamento das diferentes soluções de circularidade para resíduos têxteis; (ii) avaliação do nível de maturidade das soluções; (iii) proposição das soluções mais adequadas aos resíduos têxteis pré-consumo da indústria. Observou-se que a reciclagem, o upcycling e o cascading podem ser soluções interessantes, contudo é necessário observar o tamanho da peça do resíduo, seu volume e o valor final do produto a fim de decidir a estratégia ótima.

Palavras chave: economia circular; resíduo têxtil pré-consumo; indústria calçadista; TRL 


\section{INTRODUÇÃO}

O setor calçadista é economicamente relevante no Brasil, produzindo 763,7 milhões de pares em 2020, correspondendo a R\$ 20.981 milhões (ABICALÇADOS, 2021). O modelo econômico vigente, baseado numa lógica linear de produção, consumo e descarte de produtos, gera impacto ambiental negativo, como perda da biodiversidade, poluição da água e solo, esgotamento de recursos naturais (WWF, 2018). O modelo linear também pode gerar impactos negativos sociais, como exploração de trabalhadores, a exemplo do desabamento do edifício Rana Plaza por desatendimento de normas de segurança (ILO, 2020).

A indústria calçadista tem produzido esforços para redução do impacto dos resíduos na cadeia produtiva de maneira incremental e segmentada (FRANCISCO et al., 2014). Contudo, estima-se que em 2020 foram gerados cerca de 7.556 toneladas de resíduo têxtil na indústria calçadista brasileira, revelando a importância de estudos centrados neste problema. Dada a relevância da indústria calçadista no RS, responsável por $21,2 \%$ dos pares nacionais (ABICALÇADOS, 2021), soluções para os resíduos têxteis são fundamentais.

Nessas circunstâncias, a Economia Circular (EC) ganha importância como suporte ao desenvolvimento de políticas públicas e de negócios (KORHONEN et al., 2018), oferecendo uma alternativa para se repensar o modo de produzir e consumir de maneira sustentável (TSENG et al., 2020). Publicação da Ellen MacArthur Foundation (EMF, 2015) propõe diferentes estratégias para aumento do ciclo de vida dos materiais e reaproveitamento do que seria considerado resíduo. Os pesquisadores do Núcleo de Inteligência para Projetos e Sistemas - CNPq (NIProS - dgp.cnpq.br/dgp/espelhogrupo/664428) têm se dedicado a responder perguntas como: quais são as estratégias circulares mais adequadas aos resíduos têxteis pré-consumo da indústria calçadista?

No contexto da EC, importa primariamente em qualquer segmento produtivo desenvolver soluções inovadoras que sequer levem à geração de resíduos. No entanto, para os processos produtivos lineares existentes, também podem ser pensadas soluções “end of pipe”. Estas não eliminam a causa do problema na sua raiz, mas minimizam os impactos dos produtos que ainda geram resíduos. Assim, o objetivo deste artigo é encontrar as soluções mais adequadas aos resíduos têxteis pré-consumo da indústria calçadista. Para isso, a metodologia é baseada numa pesquisa Desk por soluções circulares que possam ser aplicadas ao resíduo têxtil préconsumo da indústria calçadista, classificado-as posteriormente segundo níveis de maturação tecnológica - Technology Readiness Level (TRL). 
Esta publicação faz parte de uma pesquisa mais abrangente que visa desenvolver sistema circular têxtil interorganizacional em Porto Alegre. Este estudo contribui para o estado da arte, propondo uma metodologia que envolve pesquisa Desk associada à métrica TRL. Além disso, há contribuição para o estado da prática, pois poucos artigos enfatizam a EC dos calçados, especialmente em relação aos componentes têxteis.

\section{REVISÃO TEÓRICA}

Esta seção permite um maior aprofundamento nos conceitos base desta pesquisa, incluindo a conceitualização da EC e dos resíduos da indústria calçadista.

\subsection{Economia circular}

Segundo a EMF (2015), a EC tem como princípio base o fechamento de ciclos no fluxo de materiais, i.e., um sistema fechado que minimiza desperdícios, resultando na eliminação de inputs de novos recursos, de resíduos e de emissões no sistema (GEISSDOERFER et al., 2017). É uma alternativa de produção complementar ou substituta à economia linear tradicional, na qual se mantém os recursos em uso pelo maior tempo possível, extrai-se o valor máximo deles enquanto em uso, depois recupera-se e regenera-se produtos e materiais ao final de cada vida útil. Existem quatro níveis de implementação da EC: (a) governamental, regional, nacional ou urbano; (b) interorganizacional; (c) organizacional; e (d) produto (WBCSD, 2018).

De acordo com a EMF (2015), a EC pode ser desmembrada em dois ciclos, apresentados na Figura 1: o ciclo biológico, que representa o fluxo de materiais renováveis biodegradáveis; e ciclo técnico, que apresenta o fluxo de materiais finitos. Quanto mais fechado o ciclo, melhor preserva o valor do material. Além disso, a EC baseia-se em três princípios: (i) eliminação de resíduos e da poluição desde o princípio; (ii) manutenção dos produtos e dos materiais em uso; (iii) regeneração dos sistemas naturais. 


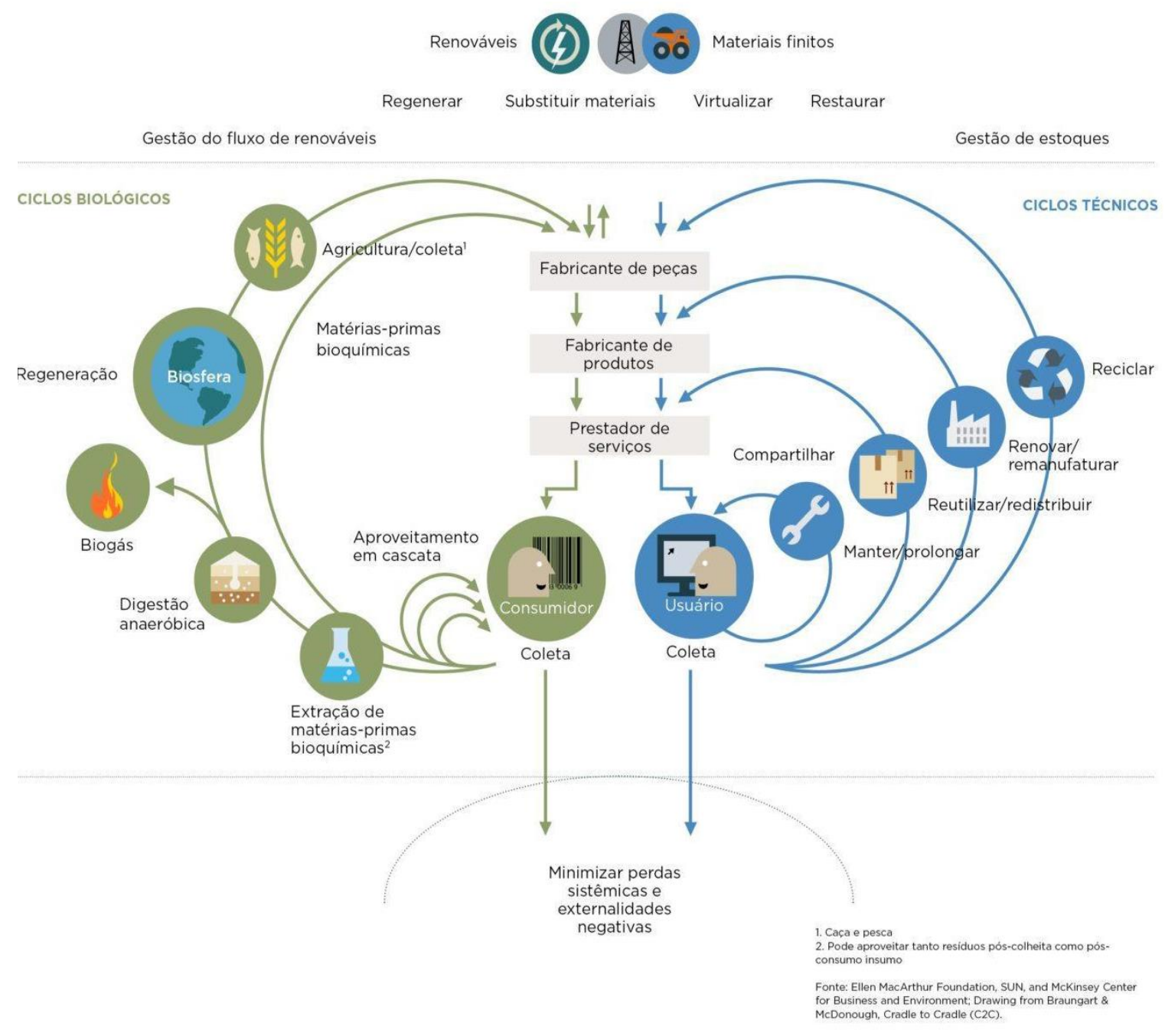

FIGURA 1 - Diagrama borboleta para a EC. Fonte: EMF, 2015, p. 6

No caso dos resíduos têxteis, pode-se observar as seguintes estratégias: compartilhamento de roupas, upcycling e reciclagem. O compartilhamento de roupas se caracteriza como uma estratégia de servitização. Já o upcycling é definido como processo de reparo, reutilização, reaproveitamento, recondicionamento e remanufatura de produtos e materiais que seriam descartados, mantendo ou aumentando o valor original do item (BHATT; SILVERMAN; DICKSON, 2018; SINGH et al., 2019). É uma prática de recriação de produtos para aumento da qualidade e valor de resíduos e materiais usados por meio de processos de transformação, reaproveitamento e remodelagem (SUNG, 2015). O upcycling difere da reciclagem pois a reciclagem é uma operação de recuperação em que materiais são reprocessados em produtos, materiais ou substâncias para os mesmos fins ou fins diferentes (PARLAMENTO EUROPEU E O CONSELHO DA UNIÃO EUROPEIA, 2008), podendo haver perda de parte do seu valor (SINGH et al., 2019). Por exemplo, no caso de tecidos de algodão, o comprimento das 
fibras diminui no processo de reciclagem, prejudicando sua qualidade e reutilização (ÜTEBAY; ÇELIK; ÇAY, 2019). Enquanto isso, ao utilizar-se a estratégia do upcycling, o resíduo de algodão seria usado na criação de uma nova peça, valorizando características intrínsecas do material para aumentar seu valor. Por isso, muitas vezes se refere à reciclagem tradicional como downcycling. Ao observar-se o diagrama de fluxo de materiais na EC, Figura 1 (EMF, 2015), percebe-se que o upcycling não aparece de forma explícita. O ciclo da reciclagem poderia ser dividido em dois ciclos concêntricos: upcycling (mais interno) e downcycling (mais externo), enfatizando diferenças de manutenção de valor dos materiais e de uso de recursos necessários em cada um deles.

A EC encontra desafios para implementação devido às limitações tecnológicas, à inércia institucional e ao fato de preferências dinâmicas do consumidor restringirem a escalabilidade desses negócios (PAL; GANDER, 2018). Atualmente há uma tendência de aumento da consciência sobre a importância da sustentabilidade. Entretanto, Harris, Roby e Dibb (2015) ressaltam que oferecer opções de produtos sustentáveis não é suficiente para mudar o comportamento do consumidor. Especificamente em relação às vestimentas, os clientes não as compram por serem sustentáveis e apresentam conhecimento mínimo sobre o impacto ambiental da indústria da moda (LEAL FILHO et al., 2019). Isso torna-se um obstáculo para empresas buscando incorporar a EC, pois muitos modelos de negócio inovadores e sustentáveis falham em convencer sobre os benefícios dos produtos sustentáveis. Assim, a educação do consumidor é necessária para incentivar um comportamento individual mais sustentável (TODESCHINI et al., 2017).

Apesar dessas dificuldades, negócios centrados na EC alcançam lucratividade e crescimento por meio do aumento da eficiência de produção, mitigação de riscos e de novas oportunidades de receita que exploram limitações de recursos de forma inovadora (WHALEN; WHALEN, 2020). Ademais, há maior proteção do meio ambiente graças à diminuição de resíduos (MOKTADIR et al., 2020). Segundo Rattalino (2017), é possível atingir vantagem circular e progresso econômico e sustentável tendo uma visão de longo prazo e uma abordagem disciplinada em relação à inovação voltada para sustentabilidade.

\subsection{Resíduos têxteis da economia circular}

Apesar da grande produção de calçados no Brasil, há um número limitado de publicações que abordam sustentabilidade nas cadeias calçadistas, concentrando-se genericamente na indústria 
da moda (CIASULLO; CARDINALI; COSIMATO, 2017). As publicações encontradas acerca da sustentabilidade na produção de calçados abordam especificamente o couro (MARQUES; GUEDES; FERREIRA, 2017; MOKTADIR et al., 2020), material predominante em $18,8 \%$ dos calçados brasileiros, atrás do plástico/borracha $(56,4 \%)$ e dos laminados sintéticos $(21,9 \%)$ (ABICALÇADOS, 2021). Nesse contexto, os materiais têxteis são predominantes em 2,3\% dos calçados produzidos. Contudo, esses valores não consideram as quantidades de tecido utilizadas em menor escala na confecção de um calçado. Segundo Staikos et al. (2006), materiais têxteis correspondem a 6\% do peso de um sapato típico.

O volume de resíduos têxtil pré-consumo corresponde a $25 \%$ dos recursos utilizados (RUNNEL et al., 2017). Assim, considerando que o sapato típico, peso médio de $1 \mathrm{~kg}$, possui $6 \%$ de sua composição correspondente a tecidos (STAIKOS et al., 2006) e sabendo que a produção total em 2020 foi de 377.800 .000 de pares de calçados desconsiderando chinelos (ABICALÇADOS, 2021), estima-se então que em 2020 foram gerados cerca de 7.556 toneladas de resíduo têxtil pré-consumo na indústria calçadista brasileira.

\section{METODOLOGIA}

Esta seção apresenta as etapas que foram implementadas para o desenvolvimento do estudo.

\subsection{Unidade de estudo}

Essa pesquisa foi implementada na sede administrativa e de P\&D de uma indústria calçadista localizada no Rio Grande do Sul (RS) - Brasil. A empresa possui duas fábricas para produção em larga escala no Nordeste. Além disso, nessa pesquisa foram analisadas soluções para circularidade têxtil em nível mundial, buscando as soluções que fossem mais adequadas às necessidades da indústria calçadista em questão.

\subsection{Método de trabalho}

Utilizou-se a pesquisa Desk baseando-se na pesquisa na mídia popular (KUMAR, 2013), um método que utiliza dados existentes, nesse caso, disponíveis na internet. Focou-se em sites especializados em inovação tecnológica na indústria de cirularidade têxtil. Usando o motor de busca (Google Search), os autores deste artigo pesquisaram soluções circulares para os resíduos têxteis (pré e pós-consumo) entre setembro/2020 e junho/2021. Os termos usados na busca foram: "(textile recycling innovation) OR (textile upcycling) OR (clothes sharing) OR “ (textile circularity)" . Os critérios de seleção foram: (i) utilizar resíduo têxtil como fonte de 
criação de produtos, serviços ou processos; (ii) ser uma ideia testada ou em desenvolvimento, produto comercializado, serviço ativo ou processo ativo em alguma empresa/instituição. As localizações geográficas dos negócios identificados foram mapeadas utilizando-se Google My Maps. Assim, criou-se um mapa das soluções circulares para resíduos têxteis que poderiam servir de inspiração para encontrar estratégias adequadas para empregar os resíduos pré-consumo da indústria calçadista. Finalmente, as soluções foram classificadas de acordo com seu nível de prontidão tecnológica (TRL).

O TRL é uma escala para classificação de tecnologias aplicáveis a projetos de inovação, cujo objetivo é apoiar gestores na tomada de decisões relativas aos investimentos e esforços por etapa de desenvolvimento, a fim de levar à evolução para níveis mais elevados de maturidade (ABGI Brasil, n.d.). Essa escala foi desenvolvida pela NASA na década de 1970, focando em sistemas espaciais. Contudo, pode ser utilizada para projetos de pesquisa, desenvolvimento e inovação de outros setores, tendo sido consolidada pela ISO 16290:2013 (VALENTE, 2020).

A ABNT realizou a adaptação da norma internacional através da NBR ISO 16290:2015. Os níveis de maturidade de 1 a 9 são descritos no Quadro 1.

QUADRO 1 - Níveis do TRL. Fonte: Valente (2020)

\begin{tabular}{|c|l|}
\hline TRL & Definição do nível de maturidade \\
\hline 1 & Princípios básicos observados e reportados \\
\hline 2 & Formulação de conceitos tecnológicos e/ou aplicação \\
\hline 3 & $\begin{array}{l}\text { Estabelecimento de função crítica de forma analítica ou experimental e/ou prova de } \\
\text { conceito }\end{array}$ \\
\hline 4 & Validação funcional dos componentes em ambiente de laboratório \\
\hline 5 & Validação das funções críticas dos componentes em ambiente relevante \\
\hline 6 & Demonstração de protótipo do sistema em ambiente operacional \\
\hline 7 & Demonstração de protóptipo do sistema em abiente operacional \\
\hline 8 & Sistema qualificado e finalizado \\
\hline 9 & Sistema operando e comprovado em todos os aspectos de sua missão operacional \\
\hline
\end{tabular}

A Figura 2 apresenta os níveis do TRL divididos em cinco grupos: (i) pesquisa básica; (ii) pesquisa aplicada; (iii) desenvolvimento experimental; (iv) industrialização; (v) produção e comercialização. Seu objetivo é correlacionar o TRL com as atividades de desenvolvimento 
de um projeto de pesquisa, desenvolvimento e inovação. Dois pesquisadores fizeram a análise das soluções para fins de classificação TRL.

\section{TRL E O CICLO DE VIDA DO PROJETO}

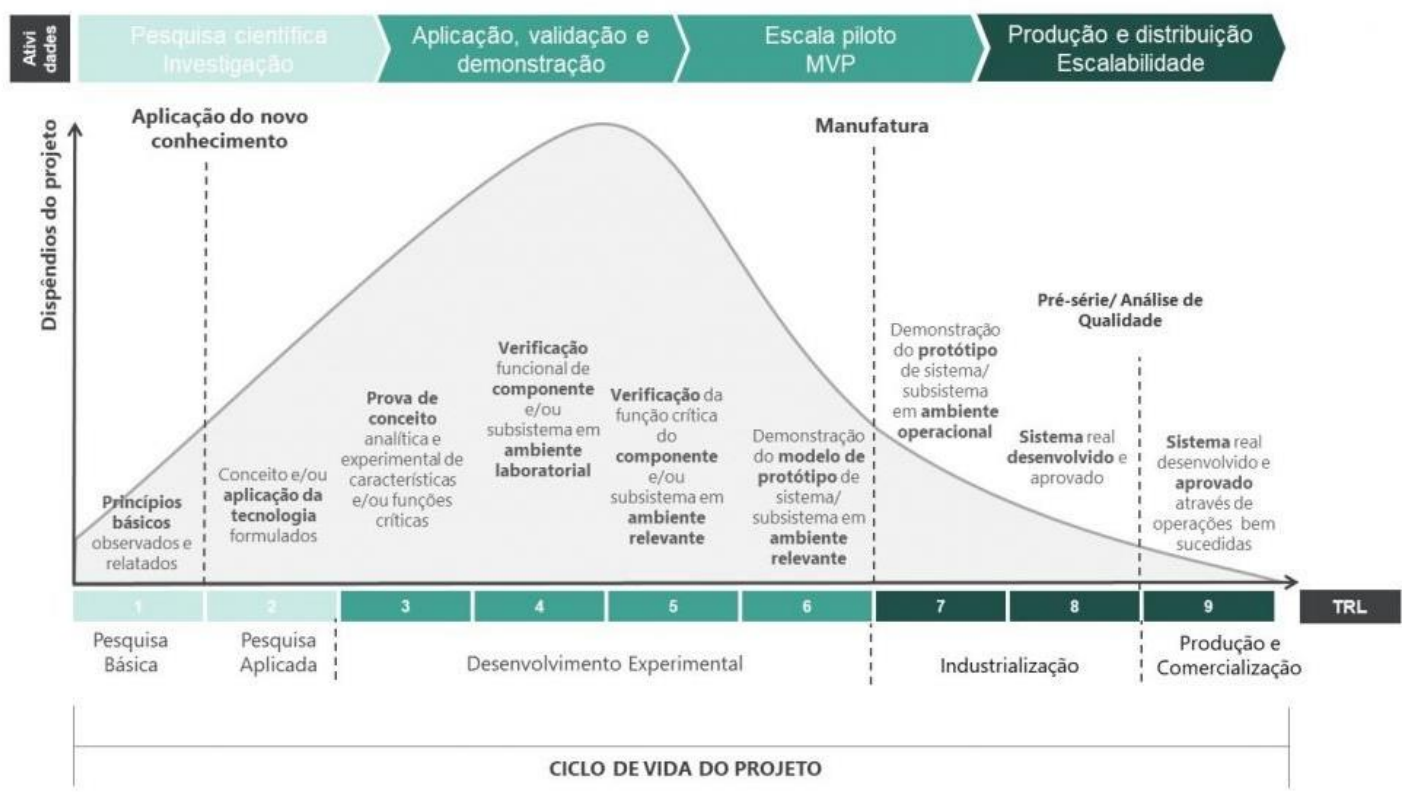

FIGURA 2 - TRL e o ciclo de vida. Fonte: ABGI Brasil (n.d.)

Usando como critérios o TRL e adequabilidade das soluções aos resíduos da indústria calçadista, foi possível selecionar as alternativas mais adequadas.

\section{RESULTADOS E DISCUSSÃO}

A revisão de literatura mostra que a indústria da moda é um dos setores mais poluentes no mundo (SHIRVANIMOGHADDAM et al., 2020). Portanto, buscou-se entender quais eram as diferentes soluções para a circularidade de materiais têxteis mapeando alternativas ao redor do mundo. O resultado pode ser visualizado no link: https://bit.ly/3pTt7Xs (Figura 3). Ao acessar o mapa, é possível ler a descrição de cada uma das soluções e sua fonte. Nota-se que um terço das soluções encontradas estão na Europa. Isso deve-se, possivelmente, ao fato de ali haver iniciativas de implementação da EC em nível governamental. Por exemplo, países como França (MINISTÈRE DE LA TRANSITION ECOLOGIQUE, 2020), Suécia (GOVERNO DA SUÉCIA, 2020) e Países Baixos (GOVERNO HOLANDÊS, 2016) possuem um plano estratégico para implementação da EC em nível nacional. 


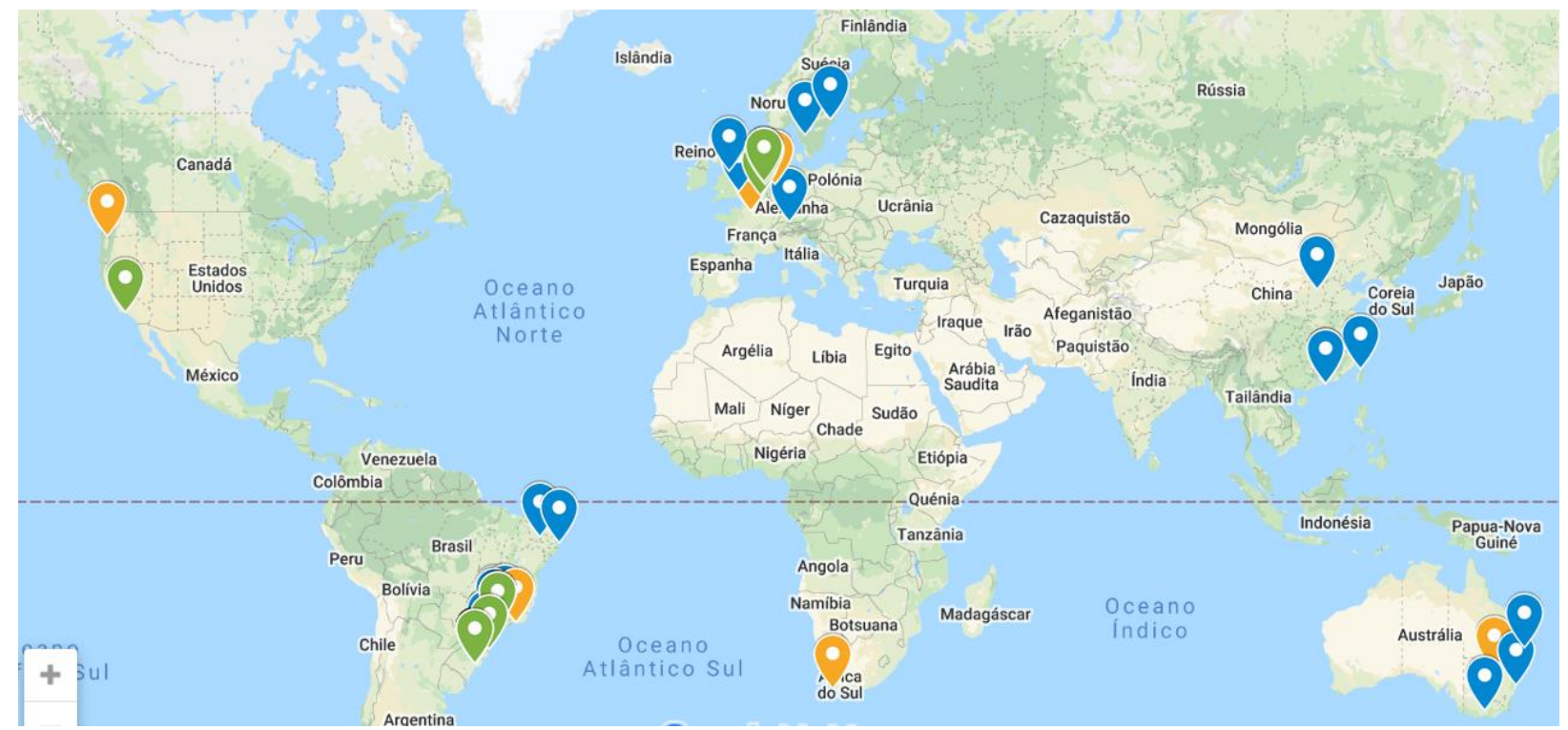

FIGURA 3 - Localização das empresas que desenvolvem soluções de circularidade no segmento têxtil em diferentes continentes: link: https://bit.ly/3pTt7Xs

Foram encontradas quarenta e oito soluções, classificadas segundo os ciclos da EMF (2015): reciclar, renovar/remanufaturar, reutilizar/redistribuir, compartilhar, manter/prolongar. Além disso, foi utilizado o conceito de cascading ("cascata"): processos que fazem parte do ciclo biológico nos quais materiais e componentes usados são utilizados para diferentes usos, extraindo ao longo do tempo a energia embutida (EMF, 2015). À medida que o valor é extraído do material, ele perde valor e é decomposto. Ademais, adicionou-se o conceito de upcycling, pois, nos ciclos da EMF (2015), descreve-se a reciclagem como o retorno ao nível da matéria-prima enquanto o upcycling diz respeito à processos de transformação, reaproveitamento e remodelagem dos resíduos para aumento da qualidade, preservando a qualidade existente no material (SUNG, 2015).

Dessa forma, usando as classificações mencionadas, elaborou-se o gráfico da Figura 4, que apresenta a composição das soluções encontradas. Algumas empresas desenvolviam soluções que se encaixavam em mais de uma classificação. A partir da análise da Figura 4, percebe-se que a maioria das soluções são de reciclagem (45\%), seguidas pelo upcycling (21\%) e cascading (14\%). É interessante notar a representatividade do upcycling, que tem atraído o interesse da indústria e da academia, acompanhado pelo aumento do número de publicações, especialmente, a partir de 2008, sendo a maior parte das pesquisas focadas na indústria da moda e tecidos (SUNG, 2015). Na pesquisa, não foram encontradas soluções do tipo renovar/remanufaturar: quando se desmonta um produto, substituindo as peças necessárias, para remontá-lo com a mesma função e qualidade do produto inicial. 


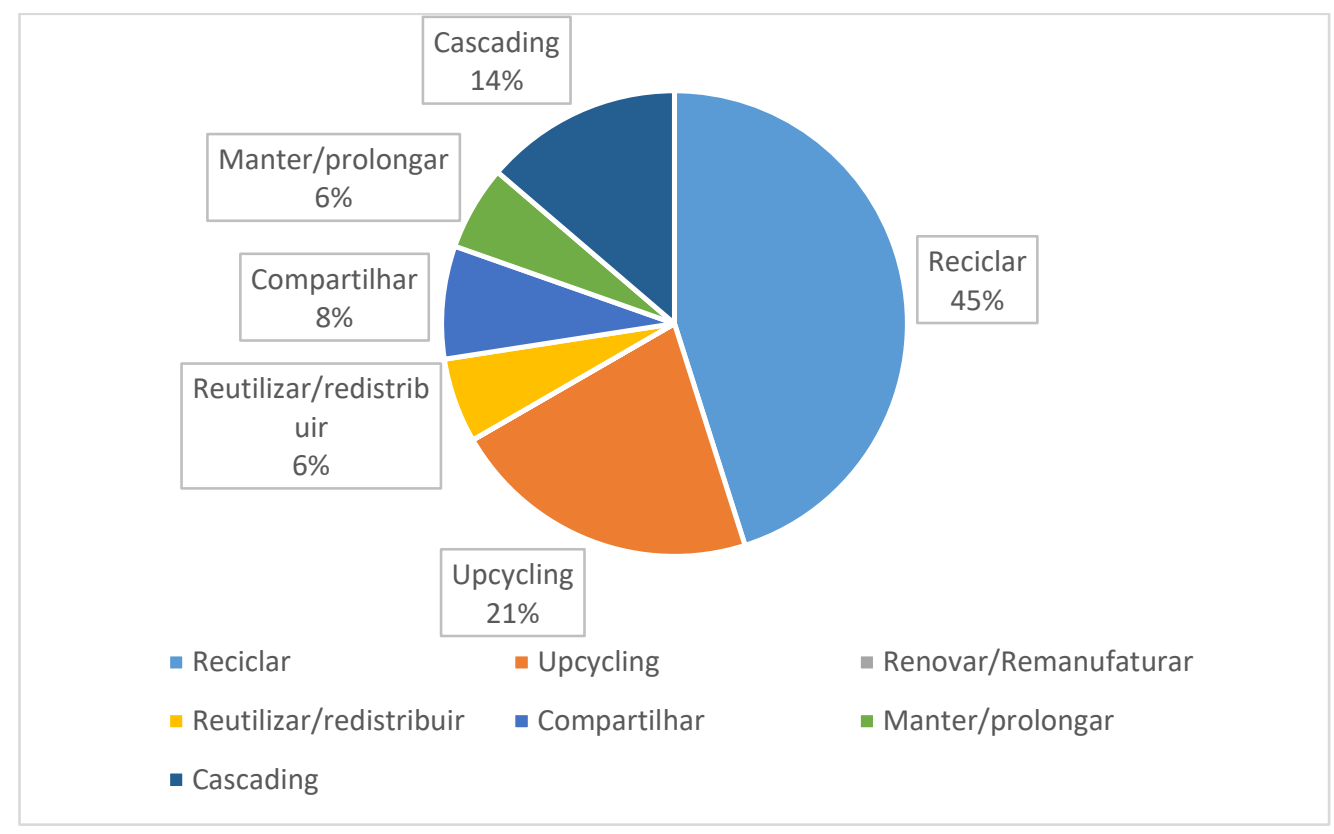

FIGURA 4 - Classificação das soluções para circularidade têxtil

Depois, as soluções foram analisadas segundo o TRL. Com isso, construiu-se a Figura 5. Pode-se observar que a maioria das soluções encontradas está no nível 9, que corresponde a "Sistema operando e comprovado em todos os aspectos de sua missão operacional", pois a maioria das soluções encontradas já estavam em fase de comercialização. Contudo, foram encontradas soluções nos níveis mais iniciais, na fase de pesquisa aplicada e desenvolvimento experimental, principalmente em relação à reciclagem de fibras naturais e sintéticas, e.g., algodão/poliéster.

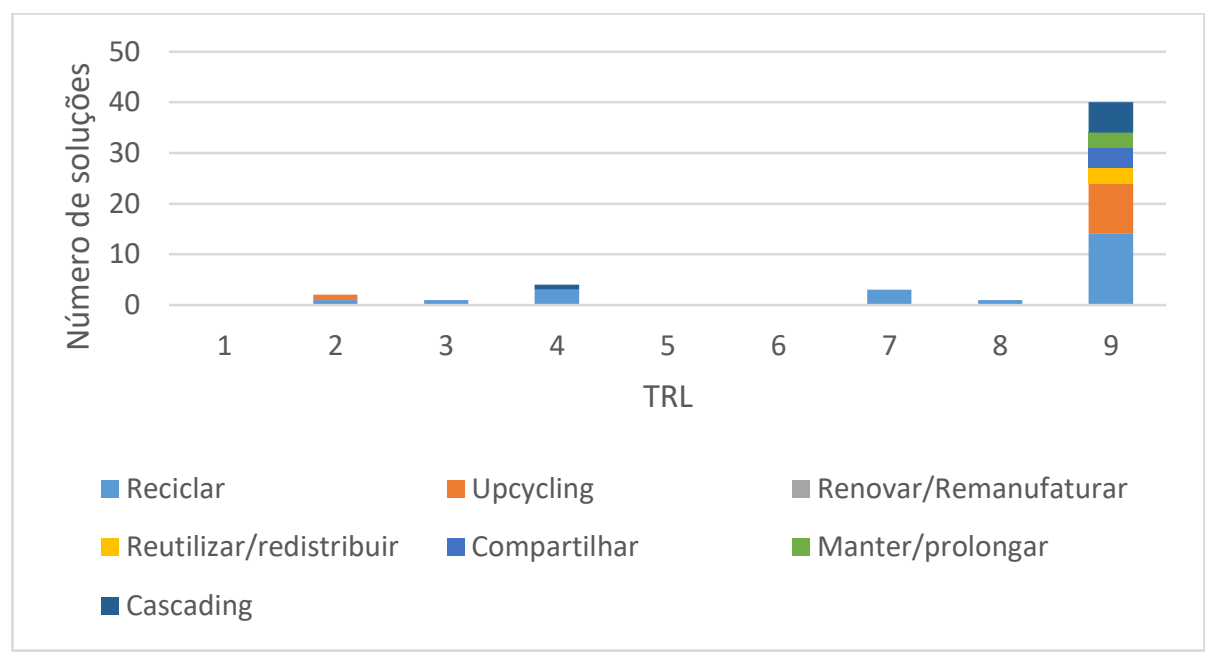

FIGURA 5 - Classificação das soluções segundo o TRL

Os resíduos têxteis pré-consumo da indústria calçadista estudada consistiam em rebarbas e finais de rolo com variedade de materiais: algodão, fibras sintéticas e misturas de fibras. Comparando as soluções encontradas e as características dos resíduos têxteis pré-consumo da empresa estudada, constata-se o seguinte: 
- Reciclar: É uma solução eficiente para rebarbas considerando altos volumes. Muitas das soluções existentes possuem alto nível de maturidade, consistindo na moagem dos resíduos, seguida de fiação. Entretanto, nesse processo há perda do valor do material, pois há diminuição do comprimento das fibras. Ainda assim, existem inovações de reciclagem mecânica como The Billie (https://thebillieupcycling.com/) que propõe um processo mantendo o valor dos tecidos e sem utilização de água. Há também o desenvolvimento de soluções de separação das fibras sintéticas e naturais através de processos de dissolução das fibras sintéticas, interessante para as rebarbas contendo misturas.

- Upcycling: Para os finais de rolo, que apresentam tamanho maior, as soluções de upcycling são uma alternativa viável, pois geram produtos criativos com alto valor agregado. Em relação às rebarbas, é possível gerar produtos com esta estratégia, mas é necessário maior tempo de produção. As soluções costumam ter um alto nível de maturidade.

- Cascading: As soluções de cascading possuem alto nível de maturidade, mas apresentam graus variados de valor agregado. Por exemplo, isolantes térmicos com resíduos possuem alto nível de degradação e menor valor agregado. Contudo, foram encontradas também soluções como painéis de parede e blocos para decoração que possuem maior valor agregado. Essas soluções seriam interessantes para as pequenas rebarbas, pois absorvem grandes quantidades e preservam o valor estético do material.

- Reutilizar/redistribuir: para os finais de rolo, soluções como a do Banco de Tecido (https://bancodetecido.com.br/) seriam interessantes pois redirecionam resíduos que não foram utilizados para pessoas que necessitam deles, evitando a formação de estoque morto. Já existem soluções deste tipo com alto nível de maturidade no mercado.

As soluções de compartilhamento não se adequam ao caso dos resíduos pré-consumo da indústria, pois não são produtos que podem ser utilizados por outras pessoas.

\section{CONCLUSÃO}

Este artigo teve como tema a circularidade de resíduos têxteis pré-consumo do segmento calçadista. Seu objetivo foi encontrar as soluções mais adequadas aos resíduos têxteis pré- 
consumo da indústria calçadista. A metodologia adotada gerou um mapa localizando diversas soluções de circularidade, as quais foram classificadas de acordo com os ciclos da EMF (2015) e com o nível de maturidade TRL.

Observou-se que países europeus têm investido em soluções governamentais para implementação da EC, auxiliando no desenvolvimento de novas tecnologias. Considerando as necessidades da indústria calçadista, priorizam-se as soluções com maior nível de maturidade, por estar disponível com maior prontidão para implementação no sistema circular nascente. A premissa é que adotando soluções que estão prontidão e já são comercializadas, haveria uma demanda puxada, diminuindo o risco de desenvolver um produto que ficasse parado no estoque sem ser vendido. Notou-se que a reciclagem possuía o maior número de soluções, sendo a maioria com alto nível de maturidade. A reciclagem e o cascading apresentaram-se como soluções adequadas para as rebarbas da indústria calçadista, considerando o volume e o tamanho das peças. Contudo, frequentemente há perda do valor do material. O upcycling também poderia ser utilizado, principalmente para o final de rolo, considerando o aumento do valor agregado. Considerando as rebarbas, também seria possível implantar o upcycling, mas demandaria mais tempo de trabalho. Soluções de reutilizar/redistribuir, como o Banco de Tecidos, mostraram-se positivas para os finais de rolo, a fim de evitar estoque morto. $\mathrm{O}$ objetivo do artigo foi parcialmente alcançado, pois esse estudo permite uma visão global das soluções circulares, contudo seriam necessáriso experimentos para observar os resultados da aplicação in loco de tais soluções na indústria calçadista.

Entende-se que a proposta metodológica proposta neste trabalho é uma contribuição interessante para a área de sistemas circulares e EC, especialmente quando se pretende dar destino a resíduos (ainda numa perspectiva de solução de "fim de tubo"). A proposta associa iniciativas/soluções já desenvolvidas para um dado resíduo, à semelhança de uma análise de benchmarking, com a métrica TRL, visando buscar referências para um dado tipo de resíduo. Em trabalhos futuros, seria interessante fazer uma pesquisa mais próxima aos laboratórios a fim de apurar inovações em desenvolvimento que ainda não foram divulgadas.

\section{REFERÊNCIAS}

ABGI Brasil. TRL: Recursos financeiros por níveis de maturidade tecnológica. ABGI Brasil. Disponível em: https://brasil.abgi-group.com/radar-inovacao/artigos-estudos/trl-recursos-financeiros-por-niveis-dematuridade-tecnologica/. Acesso em: 16 jun. 2021.

ABICALÇADOS. Relatório anual de atividades 2020. Abicalçados, 2021. Disponível em: <http://abicalcados.com.br/publicacoes/relatorio-anual>. Acesso em: 15 mai. 2021. 
BHATT, D.; SILVERMAN, J.; DICKSON, M. Consumer interest in upcycling techniques and purchasing upcycled clothing as an approach to reducing textile waste. International Journal of Fashion Design, Technology and Education, v. 12, n. 1, p. 118-128, 2018.

CIASUllO, M.; CARDINALI, S.; COSIMATO, S. A strenuous path for sustainable supply chains in the footwear industry: A business strategy issue. Journal of Global Fashion Marketing, v. 8, n. 2, p. 143-162, 2017.

ELLEN MACRTHUR FOUNDATION. Rumo à economia circular: O racional de negócio para acelerar a transição. Ellen MacArthur Foundation, 2015. Tradução de: Towards a circular economy: business rationale for an accelerated transition, $2015 . \quad$ Disponível em: <https://www.ellenmacarthurfoundation.org/publications/towards-a-circular-economy-business-rationale-for-anaccelerated-transition>. Acesso em: 16 jun. 2020.

FRANCISCO, G. et al. Geração de resíduos ao longo da cadeia calçadista: uma discussão a partir o mapeamento da literatura In: ENCONTRO INTERNACIONAL SOBRE GESTÃ̃ EMPRESARIAL E MEIO AMBIENTE ENGEMA. 2014. FEA/USP.

GEISSDOERFER, M. et al. The Circular Economy - A new sustainability paradigm? Journal of Cleaner Production, v. 143, pp. 757-768, 2017

GOVERNO DA HOLANDA. A Circular Economy in the Netherlands by 2050. 2016. Disponível em: https://www.government.nl/topics/circular-economy/documents/policy-notes/2016/09/14/a-circulareconomy-in-the-netherlands-by-2050. Acesso em: 12 jun. 2021.

GOVERNO DA SUÉCIA. Circular economy: Strategy for the transition in Sweden. 2020. Disponível em: https://www.government.se/4ad42c/contentassets/d5ab250cf59a47b38feb8239eca1f6ab/circular-economy-strategy-for-the-transition-in-sweden. Acesso em: 12 jun. 2021.

HARRIS, F.; ROBY, H.; DIBB, S. Sustainable clothing: challenges, barriers and interventions for encouraging more sustainable consumer behaviour. International Journal of Consumer Studies, v. 40, n. 3, p. 309-318, 2015.

ILO. The Rana Plaza Accident and its aftermath. Disponível em: <http://www.oit.org/global/topics/geip/WCMS_614394/lang--en/index.htm>. Acesso em: 6 set. 2020.

KORHONEN, J. et al. Circular economy as an essentially contested concept. Journal of Cleaner Production, v. 175 , p. 544-552, 2018.

KUMAR, V. 101 design methods. Hoboken, N.J.: Wiley, 2013.

LEAL FILHO, W. et al. A review of the socio-economic advantages of textile recycling. Journal of Cleaner Production, v. 218, p. 10-20, 2019.

MARQUES, A.; GUEDES, G.; FERREIRA, F. Leather wastes in the Portuguese footwear industry: new framework according design principles and circular economy. Procedia Engineering, v. 200, p. 303-308, 2017.

MINISTÈRE DE LA TRANSITION ÉCOLOGIQUE. L'économie circulaire. 2020. Disponível em: https://www.ecologie.gouv.fr/leconomie-circulaire. Acesso em: 14 jun. 2021

MOKTADIR, M. et al. Circular economy practices in the leather industry: A practical step towards sustainable development. Journal of Cleaner Production, v. 251, p. 119737, 2020.

PAL, R.; GANDER, J. Modelling environmental value: An examination of sustainable business models within the fashion industry. Journal of Cleaner Production, v. 184, p. 251-263, 2018.

PARLAMENTO EUROPEU E O CONSELHO DA UNIÃO EUROPEIA. Directiva 2008/98/CE do Parlamento Europeu e do Conselho de 19 de Novembro de 2008 Relativa aos Resíduos e que Revoga Certas Directivas.

RATTALINO, F. Circular advantage anyone? Sustainability-driven innovation and circularity at Patagonia, Inc. Thunderbird International Business Review, v. 60, n. 5, p. 747-755, 2017.

RUNNEL, A. et al. The Undiscovered Business Potential of Production Leftovers within Global Fashion Supply Chains: Creating a Digitally Enhnaced Circular Economy. Reverse Resources, ago. 2017. 
SHIRVANIMOGHADDAM, K. et al. Death by waste: Fashion and textile circular economy case. Science of The Total Environment, v. 718, p. 137317, 2020.

SINGH, J. et al. Challenges and opportunities for scaling up upcycling businesses - The case of textile and wood upcycling businesses in the UK. Resources, Conservation and Recycling, v. 150, p. 104439, 2019.

STAIKOS, T. et al. End-of-Life Management of Shoes and the Role of Biodegradable Materials. 2006. Disponível em: https://www.researchgate.net/publication/229015996_End-of life_management_of_shoes_and_the_role_of_biodegradable_materials . Acesso em: 15 out. de 2020.

SUNG, K. A Review on Upcycling: Current Body of Literature, Knowledge Gaps and a Way Forward. In: INTERNATIONAL CONFERENCE ON ENVIRONMENTAL, CULTURAL, ECONOMIC AND SOCIAL SUSTAINABILITY. Veneza: 2015, v. 17 No. 4, pp 28-40.

TODESCHINI, B. et al. Innovative and sustainable business models in the fashion industry: Entrepreneurial drivers, opportunities, and challenges. Business Horizons, v. 60, n. 6, p. 759-770, 2017.

TSENG, M. et al. Circular economy enables sustainable consumption and production in multi-level supply chain system. Resources, Conservation and Recycling, v. 154, p. 104601, 2020.

ÜTEBAY, B.; ÇELIK, P.; ÇAY, A. Effects of cotton textile waste properties on recycled fibre quality. Journal of Cleaner Production, v. 222, p. 29-35, 2019.

VALENTE, M. Technology Readiness Level (TRL): conheça o framework de confiabilidade em projetos da NASA. CERTI. 2020. Disponível em: https://certi.org.br/blog/trl-desenvolvimento-projetos/. Acesso em: 16 jun. 2021 .

WBCSD. Circular Metrics: Landscape Analysis. 2018.

WHALEN, C.; WHALEN, K. Circular Economy Business Models: A Critical Examination. Journal of Economic Issues, v. 54, n. 3, p. 628-643, 2020.

WWF. Living Planet Report - 2018: Aiming Higher. Gland, Suíça: Grooten, M.; Almond, R.E.A., 2018. Acesso em: 7 set. 2020. 